\title{
LA RÉÉCRITURE D’UNE ESPÈCE EN VOIE DE DISPARITION : « LE DERNIER GODOT »
}

Georgeta-Elena Miron, Université « Stendhal », Grenoble 3, France

En 1987, deux ans avant la mort de Samuel Beckett, Matéi Visniec, un tout jeune dramaturge de Roumanie, reprend et réécrit En attendant Godot. Dans cette nouvelle pièce, le personnage Godot a la « chance » inouïe de rencontrer son créateur et élucider son absence de la pièce d'origine. Écrite en 1948 et créée en 1953 au Théâtre de Babylone, la pièce En attendant Godot signe le début de la grande carrière de Beckett, puisqu'elle est vite considérée comme la référence dramaturgique de sa génération. La rencontre artistique entre Visniec et Beckett s'est faite à travers En attendant Godot : « J'ai lu d'un seul trait cette pièce qui ne ressemblait à aucune autre pièce lue auparavant. Ce fut une révélation, le commencement d'un grand amour. J'ai découvert, à travers Beckett, ma propre identité, j'ai découvert un langage, une façon de protester, une famille d'esprit dont je faisais partie, enfin presque tout... $\gg^{1}$

A l'époque de la répression communiste soviétique, le texte beckettien constitue un acte de libération socio-politique qui initie le jeune écrivain roumain à «respirer une alternative à l'idéologie officielle. C'était comme plonger dans la liberté même de dire NON. $»^{2}$ Dans cette perspective, la pièce Le dernier Godot se veut une manière de rendre un hommage à l'auteur que Visniec $a$, tant de fois, pris pour étalon.

\section{Godot : de I'attente éternelle au rendez-vous ultime}

Les titres - symboles opposés

Le processus de la réécriture s'annonce clairement depuis le titre, car Le dernier Godot reprend le nom propre Godot de la pièce d'origine. Composé de la racine « God » et du suffixe diminutif « -ot », le nom désigne probablement une identité providentielle et familière à la fois. Le nom du personnage devient, inévitablement, le centre de la pièce et avec cela, le premier point susceptible d'intéresser la problématique de la réécriture.

En attendant Godot se transforme sous la plume de Visniec en Le dernier Godot. Tandis que Beckett accrochait au nom du personnage le verbe au gérondif, Visniec préfère éliminer complètement une quelconque action comprise dans la présence d'un verbe. Stylistiquement, le verbe beckettien suggère une action de durée, interminable.

L'attente interminable déclenchée par Beckett avec le titre de sa pièce prendra fin en quelque sorte chez Visniec. Il invente pour ce faire le dernier Godot de son espèce - comme s'il y en avait eu plusieurs... Dans le titre de Visniec il n'y a pas d'action, pas d'attente, mais une réécriture définitive et un constat ultime : comme si l'auteur voulait en finir avec ce personnage-fantôme qui a hanté les scènes des quatre coins du monde... Le titre de la pièce annonce essentiellement la fin d'une attente éternelle du personnage beckettien, que j'appellerais inhabité ... Visniec semble considérer le Godot beckettien comme victime de sa nature abstraite,

\footnotetext{
${ }^{1}$ Matéi Visniec, Le Dernier Godot, la Compagnie Pli Urgent aux Éditions du Cosmogone, 1996, p. 47

2 L'intervention est reproduite partiellement et suit la pièce Le Dernier Godot, la Compagnie Pli Urgent aux Éditions du Cosmogone, 1996, p. 48.
} 
et veut lui donner un corps pour se rendre à ses rendez-vous éternels... Mais le seul inconvénient vient de ce corps même, car il entraîne la perte de l'immortalité. Godot, l'unique, l'abstrait, la quintessence du conceptuel qui peut être tout et rien à la fois, se heurte avec Visniec à sa dernière version... le dernier Godot. Cette sentence marque la fin d'une époque, la possible mise à mort d'un personnage devenu mythe sans lui... (?)

Les didascalies - comment raconter I'histoire?

Les didascalies de la pièce beckettienne indiquent la présence de cinq personnages: Estragon, Vladimir, Lucky, Pozzo et Un jeune garçon. Pour le lecteur, il est clair que le personnage qui apparaît dans le titre ne fait pas partie de la distribution. Pour le public, comme pour les personnages de la pièce, Godot accomplira son rôle sur scène suivant le pacte inséré dans le titre.

Dans Le dernier Godot, les deux personnages distribués sont : Godot et Samuel Beckett. Si Visniec réécrit le titre, au niveau du choix des personnages, il se donne la liberté d'inventer, de transformer, d'apporter de I'originalité. C'est avec la réécriture de Visniec que Godot arrive enfin à jouer du théâtre d'après les règles classiques, mais pas tout à fait... car il a décroché presque un second rôle, une seconde chance. Beckett devient, à son tour, personnage et intègre le monde abstrait de la fiction. Visniec arrive à faire de la littérature une matière unique, en ajoutant à la fiction dramaturgique un grain de réalité historique. Les mises en abyme, les transfigurations, les transmutations tiennent de l'art d'écrire de Matéi Visniec et elles s'ajoutent à son langage simple, poétique et savoureux.

Les didascalies d'ouverture situent l'action à l'extérieur, sur la route (EAG) ou dans la rue (LDG). Les personnages beckettiens sont isolés en pleine campagne; chez Visniec, ils se situent dans la cité. Tandis qu'Estragon est «assis sur une pierre »(EAG, p.9), Godot, quant à lui, «est assis sur le bord du trottoir » (LDG, p.13). Les deux personnages ont en commun des particularités concentrées autour de leurs pieds. Si Estragon est assis, c'est parce qu'il essaie à plusieurs reprises d'enlever sa chaussure, tandis que Godot, «un homme maigrichon» (LDG, p. 13), pend ses «pieds dans le ruisseau » (LDG, p. 13) . L'action accomplie par Estragon est répétitive est représentée de manière ludique: «Même jeu. » (EAG, p.9) La didascalie s'empreigne de subjectivité de par sa qualité expressive habituellement rattachée à une narration. Visniec saisit cette nouvelle manière de se libérer des formes anciennes de composition et reprend le jeu fascinant de l'adresse narrative que son prédécesseur avait initiée pour son lecteur et seulement pour lui. Nous le retrouvons ainsi en train de « raconter » dans les didascalies : « De quelque part, d'un endroit invisible, on entend une porte qui s'ouvre, une bagarre sourde et un deuxième homme maigrichon (mais décemment habillé) est jeté sur le trottoir. Ainsi qu'on le verra plus tard, ce deuxième homme maigrichon est Samuel Beckett lui-même. Le personnage roule presque sur Godot. » (LDG, p.13) Quelques énumérations, des approximations, des parenthèses explicites et souvent contrecarrant l'opinion possible du lecteur, des anticipations temporelles, narratives, ce n'est qu'une partie de l'ensemble des techniques qui se retrouvent chez Visniec. 
Avant I'arrivée inopinée de Beckett, une poubelle tient compagnie à Godot, qui «..fume, l'air triste, sans pensée. » (LDG, p.13) Les actions des personnages sont réduites au minimum dès le début: Estragon essaie sans succès d'enlever sa chaussure, Godot passe son temps à fumer, la tête vide et l'apparence triste. Une fois le partenaire de jeu entré en scène, la solitude est comblée, mais c'est là que le drame de l'humanité devient perceptible : «ESTRAGON. - Rien à faire. » (EAG, p.9).

\section{Godot - personnage controversé}

Nous nous pencherons tout d'abord sur le personnage Godot, tel qu'il apparaît dans la pièce beckettienne, pour établir ensuite les corrélations avec la réécriture de Visniec.

\subsection{La promesse d'un avenir meilleur}

L'unique rencontre entre Vladimir, Estragon et Godot serait I'occasion pour les deux protagonistes de lui demander quelque chose, mais rien de très précis, « une sorte de prière », « une vague supplique » (p. 23).

Ce premier tableau ne met pas un visage sur le personnage Godot, mais nous aide tout de même à lier Godot aux autres héros de la pièce. Ils se trouvent subjugués dans leur rapport avec Godot, mais ils sont captifs de la même manière du milieu théâtral, de la scène et ils en ont bien conscience :

« ESTRAGON. - Quel est notre rôle là-dedans ? [...]

VLADIMIR. - Notre rôle ? Celui du suppliant. » (p. 24)

Le sens du mot «suppliant » est rapporté d'une part à leur «supérieur » Godot, et d'autre part c'est une référence à leur statut de personnages - brisant en conséquence le pacte fictionnel. Piégés dans leurs rôles et dans l'attente interminable, Vladimir et Estragon espèrent tout de même en un avenir meilleur, que seul Godot saurait leur apporter.

\subsection{Le visage du quiproquo 3}

L'impossibilité de donner un corps à Godot conduit à des confusions, à des malentendus. L'arrivée des deux autres personnages, Pozzo et Lucky, entraîne malencontreusement l'euphorie de Vladimir et Estragon, qui pensent enfin accueillir Godot. La confusion est vite dissipée, mais le doute s'installe quand Vladimir et Estragon sont incapables de reconnaître son visage.

\footnotetext{
${ }^{3}$ La technique du quiproquo était très utilisée dans le théâtre, surtout par Molière, afin de obtenir des scènes comiques. Dans la pièce beckettienne nous pouvons noter un certain humour déclenché au moment de l'entrée en scène de Pozzo et Lucky. (p. 29)
} 
Entre hésitation et maladresse, fatigue et obscurité, comment se retrouver et comment trouver l'autre ? Car le lendemain, dans le deuxième acte, Estragon est à nouveau mis en difficulté devant le retour de Pozzo et Lucky.

Attribuée sans discernement donc, l'image de Godot prend à chaque fois le visage du quiproquo. Le processus est réitéré mécaniquement en boucle et il concerne un cadre plus général de la pièce. Il s'agit de la dépersonnalisation de l'être devant I'autrui, que cela soit par le changement de nom (Vladimir s'appelle aussi Didi ou Monsieur Albert), ou par l'incapacité de reconnaître son interlocuteur ou se reconnaître soi-même. Perte d'identité, oubli, confusion, Beckett mine I'humanité en lui montrant son vrai visage...et cette fois ce n'est pas du quiproquo...

\subsection{Godot : I'imperceptible}

A la fin de chaque acte, Le Garçon, le cinquième personnage de la pièce, apparaît et transmet à Monsieur Albert, alias Vladimir, le même message: qu'il ne peut pas passer les voir ce soir mais qu'il passera certainement le lendemain soir. Tout comme les passages cycliques et aphasiques de Pozzo et Lucky, le message apporté par le Garçon a quelque chose d'un rituel nocturne. Le Garçon ne se souvient jamais d'avoir déjà rencontré les deux personnages, et pourtant il y revient chaque soir.

A la fin du deuxième acte, nous apprenons quand même que Monsieur Godot a une barbe blanche, qu'il bat le frère du messager, qu'il a des brebis mais qu'il ne fait rien de la journée. Le peu d'éléments de son portrait converge vers une hypothèse qui semblait se profiler au tout début, avec la prière des protagonistes. A ce postulat s'ajoutent : la barbe blanche de Godot, le fait qu'il soit entouré d'enfants, son absence physique qui est compensée par des signes de son existence, sa manière imperceptible de parler à ceux qui veulent l'écouter.

Le portrait qui se tisse peut sembler reconnaissable par tout le monde... mais personne ne peut dire le connaître véritablement..

\section{La revanche d'un héros}

Nous avons observé tout au long de ces trois sous-parties, les différentes étapes dans la construction d'un personnage à travers les autres. Avec Matéi Visniec, nous allons découvrir un autre visage de Godot, celui qui demande des explications à son auteur, qui crie son mécontentement, qui veut un rôle comme les autres. En toute liberté de création et de réécriture, Godot devient personnage autant que Beckett.

\section{1 « Je joue donc j'existe »}

La rencontre entre Godot et Beckett se fait à l'extérieur du théâtre, après qu'ils ont été tous les deux jetés de la salle de spectacle. Beckett roule en sortant sur Godot et une vraie discussion se profile. Beckett joue son vrai métier d'auteur, attirant la surprise de Godot: «Formidable ! Donc tu existes. » (p. 21) Le jeu de 
perspectives fait inverser les réalités. Normalement c'était Godot celui qui n'existait pas. Mais puisque tous les deux existent, Godot y trouve l'occasion d'insulter son créateur et d'exprimer sa colère haut et fort ( Si tu veux le savoir tu m’as assez frappé ! », p. 23). L'allusion à la pièce En attendant Godot témoigne d'une mémoire affective qui survole le temps. Petit à petit, les tortures remontent à la surface et démasquent un héros humilié, vindicatif, qui proclame en se mettant debout, comme au théâtre, son identité : « Je suis Godot ! » (p. 24). Son premier numéro d'expression à l'allure de mise en abyme, de théâtre dans le théâtre, montre à quel point la nécessité de jouer est viscérale pour le héros. Son discours monte en tension, le sentiment de vengeance I'anime : «Malheureux! Maintenant tu vas payer pour tout, tout, tout!» (p. 24). Ne trouvant pas sa place parmi les personnages de théâtre depuis ses débuts, Godot tente d'obtenir une explication de son auteur: «Es-ce qu'on a jamais vu un personnage qui n'apparaît pas? 》 (p. 25) L'interrogation est nette, la réponse muette. Seule la conclusion est facile à tirer : le personnage est créé pour jouer sur scène.

\subsection{Le porte-parole d'une absence...}

La prise de conscience de sa vraie nature par un personnage rime chez Visniec avec une préoccupation pour la philosophie. L'auteur, lui-même diplômé en philosophie, n'hésite pas à poser avec Godot des problèmes fondamentaux de I'humanité : «Au fond qui suis-je ? [...] Être et ne pas être, en même temps ? Voilà ce que je veux te demander, tu comprends ? » (pp. 26-27). Le Nosce te ipsum («Connais-toi toi-même ») de Socrate pousse Godot sur le chemin de la connaissance. Les réponses à la question se trouvent toujours à l'intérieur... Mais à l'intérieur de qui ? De Godot qui n'est qu'un personnage réinventé ou de Beckett qui est son créateur piégé dans une pièce ?... Une certitude domine la scène tout de même. Si Godot est un révolté, il est en même temps le porte-parole d'un fléau qui menace la société. La dégradation de l'humanité, que Beckett-auteur avait pressentie et suggérée dans ses pièces, est rendue par Visniec plus explicite encore. Son Godot peut porter plainte contre l'indifférence et le manque de communication accru de notre époque. A travers son mal d'être, le personnage demande à son créateur de justifier sa création :

« Godot - Comment est-il possible d'être et de ne pas être?

Beckett - Je ne sais pas. [...] Ça m’est venu comme ça, spontanément. » (pp. 27-28)

A travers la réponse de Beckett, c'est Visniec qui répond, car l'écriture, comme tout art, jaillit d'un processus inexplicable et complexe. Impossible donc de justifier sa création, l'inspiration n'a pas de raison d'être. Alors Godot lui demande de la refaire sa pièce :

« Godot - Glisse-moi quelque part. N'importe où... Ouvre une parenthèse... Fait quelque chose...

Beckett - Inutile. Le théâtre a fermé. » (p. 29)

Crise du personnage, crise du théâtre, Estragon dirait encore : « Rien à faire »... 


\subsection{Camarades de jeu}

Et pourtant quand toute communication semblait impossible, les deux protagonistes se mettent à parler du théâtre et ses crises, de la déshumanisation de la société, de l'individualisme, de la violence, tout autour d'un mégot récupéré des poubelles et d'une bouteille d'alcool. Et leur dialogue se tisse, en attirant des gens... et les gens se transforment en public, et ce qu'ils disent pourrait être du théâtre, même dans la rue, ou surtout dans la rue, car c'est là que commedia dell'arte est née, il y a cinq cents ans. A travers l'amitié (Godot trouve son créateur «de plus en plus sympa ») Beckett est prêt à faire le compromis d'introduire Godot dans sa pièce, mais c'est Godot qui ne l'accepte plus. L'art garde ainsi sa pureté, son intégrité et intégralité.

Sans pouvoir s'y opposer, le public se précipite autour d'eux. Godot panique (« Mon Dieu, qu'est-ce que je dois dire ? »), mais Beckett sait lui donner le ton, lui inculquer, enfin, le courage... de jouer. La fin de la pièce n'est autre chose que le début d'En attendant Godot, mot pour mot. La réécriture ne va pas jusqu'à la réinvention de l'œuvre d'origine, la pièce En attendant Godot restera pour toujours la même. Godot et Beckett sont des camarades de jeu de la même équipe, le temps d'une pièce.

\section{Conclusions : la réécriture - quel rôle joue-t-elle ?}

L'intertextualité est, d'après Gérard Genette ${ }^{4}$, la « relation de coprésence entre deux ou plusieurs textes, c'est-à-dire éidétiquement et le plus souvent, par la présence effective d'un texte dans un autre. » Sa forme explicite et littérale est la citation. La forme moins explicite est le plagiat (emprunt non déclaré, mais encore littéral). Enfin, la forme encore moins explicite et moins littérale est l'allusion (« énoncé dont la pleine intelligence suppose la perception d'un rapport entre lui et un autre auquel renvoie nécessairement telle ou telle de ses inflexions, autrement non recevable »).

Nous constatons dans le cas étudié que la première et la troisième forme d'intertextualité coexistent avec I'hypertextualité. La forme explicite, c'est-à-dire la citation, est présente dans le texte de Visniec, à la fin de la pièce. Le choix, plus que suggestif, contribue à la technique de la mise en abyme, du théâtre dans le théâtre. En ce qui concerne la deuxième forme, nous avons constaté depuis le titre et les didascalies jusqu'au langage employé, des allusions à la pièce beckettienne. Il s'agit bien d'allusion car le dramaturge innove aussi bien qu'il reproduit, en portant plus loin le symbole incarné par Godot, initié par son prédécesseur. Il prend plaisir à complexifier les psychologies, car son Godot existe à travers lui-même et commence à se connaître. Et nous nous retrouvons ainsi devant l'autre processus de l'hypertextualité (Genette).

Le dialogue des protagonistes est aussi actualisé à l'aide d'expressions contemporaines: (« gueule », « caboche », «foutre ») et devient ainsi presque réel. La force des sentiments est débordante, anime les discussions. Le personnage réécrit ne se résume plus à exécuter les consignes. Les échanges sont très vifs, authentiques. On pourrait très bien les imaginer dans notre quotidien.

\footnotetext{
${ }^{4}$ Gérard Genette, Palimpsestes, Le Seuil, coll. « Poétique », 1982
} 
Mais la réécriture a ses limites. Le langage, le rôle de Godot sont inventés de toutes pièces, tout comme I'invention d'un personnage tel que Beckett. Godot ne tient que les racines de son prédécesseur. Il n'a pas de barbe blanche, il n'est pas entouré de sa famille, il n'a pas de brebis et de serviteurs, et il ne peut pas, dans l'état où il est, promettre un avenir meilleur. Mais il est présent. Et ironique coïndidence, frustrante mise en abyme, Godot aurait été présent toujours là, à côté de Beckett, chaque soir spectateur de son propre spectacle, témoin de sa propre absence...

Avec la réécriture de Visniec, un personnage sans visage prend contour, une absence se veut comblée, un mystère résolu. Mais à travers tous ces éléments, Godot n'est plus le même... Quel impact la réécriture pourrait-elle avoir sur le personnage d'origine? Les quelques indices du portrait laissés par Beckett sont ignorés, car Visniec a un autre projet. Il attire Beckett sur scène et se libère enfin de son emprise. Sa démarche a une résonance de tragédie antique modernisée, car il «tue le père » mais sur scène. Et ce n'est pas tout, car avec I'auteur, Visniec anéantit aussi Godot. Une fois la libération d'expression accomplie et le héros désacralisé, il pourra se créer une nouvelle voie vers la création. Un autre chemin l'attendait, celui vers la France, car après avoir écrit cette pièce, Visniec dit NON au régime totalitaire de son pays et se réfugie à Paris.

Entre les deux textes, il existe une liaison évidente. Et si elle existe ce n'est pas pour faire du plagiat ou imiter une œuvre plus que complète, ce qui serait sans intérêt. Visniec se donne par la fiction la possibilité de dialoguer avec ce maître et en son nom porter plus loin encore quelques symboles de son œuvre. Sans la pièce de Visniec, on ne pourrait pas dire : «Moi, j’ai rencontré le dernier Godot ! » 